\title{
Prospective Evaluation of Unprocessed Core Needle Biopsy DNA and RNA Yield from Lung, Liver, and Kidney Tumors: Implications for Cancer Genomics
}

\author{
Mikhail T. Silk, ${ }^{1}$ Nina Mikkilineni, ${ }^{2}$ Tarik C. Silk, ${ }^{1}$ Emily C. Zabor, ${ }^{3}$ Irina Ostrovnaya, ${ }^{3}$ \\ Ari A. Hakimi, ${ }^{2}$ James J. Hsieh, ${ }^{4}$ Etay Ziv, ${ }^{1}$ Natasha Rekhtman, ${ }^{5}$ Stephen B. Solomon, ${ }^{1}$ \\ and Jeremy C. Durack $\mathbb{1}^{1}$ \\ ${ }^{1}$ Interventional Radiology Section, Department of Radiology, Memorial Sloan Kettering Cancer Center, 1275 York Avenue, New York, \\ NY 10065, USA \\ ${ }^{2}$ Department of Surgery, Urology Service, Memorial Sloan Kettering Cancer Center, 1275 York Avenue, New York, NY 10065, USA \\ ${ }^{3}$ Department of Epidemiology and Biostatistics, Memorial Sloan Kettering Cancer Center, 1275 York Avenue, New York, \\ NY 10065, USA \\ ${ }^{4}$ Division of Oncology, Department of Medicine, Washington University Medical School, USA \\ ${ }^{5}$ Department of Pathology, Memorial Sloan Kettering Cancer Center, 1275 York Avenue, New York, NY 10065, USA
}

Correspondence should be addressed to Jeremy C. Durack; durackj@mskcc.org

Received 18 July 2018; Accepted 29 October 2018; Published 10 December 2018

Academic Editor: Maria M. Picken

Copyright ( 2018 Mikhail T. Silk et al. This is an open access article distributed under the Creative Commons Attribution License, which permits unrestricted use, distribution, and reproduction in any medium, provided the original work is properly cited.

Context. Targeted needle biopsies are increasingly performed for the genetic characterization of cancer. While the nucleic acid content of core needle biopsies after standard pathology processing (i.e., formalin fixation and paraffin embedding (FFPE)) has been previously reported, little is known about the potential yield for molecular analysis at the time of biopsy sample acquisition. Objectives. Our objective was to improve the understanding of DNA and RNA yields from commonly used core needle biopsy techniques prior to sample processing. Methods. We performed 552 ex vivo 18 and 20G core biopsies in the lungs, liver, and kidneys. DNA and RNA were extracted from fresh-frozen core samples and quantified for statistical comparisons based on needle gauge, biopsy site, and tissue type. Results. Median tumor DNA yields from all 18G and 20G samples were $5880 \mathrm{ng}$ and $2710 \mathrm{ng}$, respectively. Median tumor RNA yields from all $18 \mathrm{G}$ and $20 \mathrm{G}$ samples were $1100 \mathrm{ng}$ and $230 \mathrm{ng}$, respectively. A wide range of DNA and RNA quantities (1060-13,390 ng and 370-6280 ng, respectively) were acquired. Median DNA and RNA yields from $18 \mathrm{G}$ needles were significantly greater than those from $20 \mathrm{G}$ needles across all organs $(p<0.001)$. Conclusions. Core needle biopsy techniques for cancer diagnostics yield a broad range of DNA and RNA for molecular pathology, though quantities are greater than what has been reported for FFPE processed material. Since non-formalin-fixed DNA is advantageous for molecular studies, workflows that optimize core needle biopsy yield for molecular characterization should be explored.

\section{Introduction}

Image-guided solid tumor needle biopsies are frequently the starting point for modern cancer care. The ability to genomically characterize tumors has amplified the importance of tissue biopsies for cancer treatment selection, determining eligibility for clinical trials and understanding disease progression. In recent years, the brisk pace of discoveries revealing the genetic basis for malignant transformation has empowered oncologists, enabling therapies targeting specific 
molecular aberrations [1-3]. Needle biopsies can provide material for targeted genetic mutation analysis or to assess response to treatment, obviating the need for surgical biopsy.

A high-quality, high-value biopsy is now defined by sufficient cancer cellularity for diagnosis and genomic analysis [4]. Diagnostic rates for contemporary targeted biopsies are high, but procedural practice guidelines have been slow to consider additional sampling requirements associated with molecular characterization [5]. Real-time CT, ultrasound, or MR image-guidance technologies have enabled more accurate percutaneous sampling of smaller targets [6]. However, the quantity of genetic material that can be obtained from small tumors is not easily defined due to many factors influencing biopsy yield, including normal tissue versus solid tumor cellularity and variable density of tumor nuclei per volume of tissue. Furthermore, single-site biopsies may not sufficiently portray intratumoral genetic heterogeneity [7].

Deoxyribonucleic acid (DNA) and ribonucleic acid (RNA) quantities required for a combination of routine clinical care, clinical trials, and research protocols often vary by individual institution and clinical team. Quantities sufficient for analysis will also vary in relation to the increasing number and range of molecular tests and technical advances in tissue analytics. Furthermore, several analyses of preanalytic factors related to tumor sequencing have raised concerns about low DNA and RNA yields from percutaneous tumor biopsies $[8,9]$.

Importantly, standard core biopsy processing in pathology laboratories includes formalin fixation and paraffin embedding (FFPE). All downstream diagnostic and molecular assays are generally performed on thin sections prepared by microtomy from FFPE tissue blocks. In most studies to date, DNA and RNA content in core biopsies has been analyzed from FFPE material, whereas quantities of nucleic acid in unprocessed core biopsies are not well established. The goal of this study was to assess DNA and RNA quantities obtained using widely used core biopsy techniques from different cancer types in order to facilitate planning and decision-making with regard to molecular oncology testing. Knowledge of needle biopsy sampling capabilities can be essential for patient management in the setting of either known or suspected cancer. For both patient and healthcare provider, the anticipated value of quantitative data to plan needle biopsies is a better understanding of the potential risk versus clinical benefit [10-12].

\section{Materials and Methods}

We performed an Institutional Review Board-approved prospective study of surgically resected specimens at a comprehensive cancer center with a waiver of informed consent. Biopsies were performed in a tissue procurement service facility under direct visualization within 2 hours of surgical excision using 18-gauge (18G) and 20-gauge (20G) core biopsy needles (Temno Evolution, CareFusion, Waukegan, IL). Each surgical specimen was first dissected to allow direct visualization of the tumor and surrounding normal tissues. Biopsies were acquired from a variety of locations in normal parenchyma and tumor, avoiding areas of visible necrosis, and each $2 \mathrm{~cm}$ long core needle sampling tray was visually inspected. Core specimens that did not fill at least $85 \%$ of the sampling tray were discarded. Biopsies were performed in triplicate using $18 \mathrm{G}$ and $20 \mathrm{G}$ needles for both DNA and RNA processing. Biopsy sample sizes were estimated based on the number of samples required to achieve statistical significance from a preliminary kidney biopsy cohort. Each specimen was immediately placed in a $1.7 \mathrm{ml}$ Eppendorf tube and snap frozen in liquid nitrogen. Samples were then stored in a $-80^{\circ} \mathrm{C}$ freezer until molecular extractions were performed.

2.1. DNA Extraction. DNA was extracted using a standard protocol (DNeasy, Qiagen, Venlo, Netherlands) with $4 \mu \mathrm{l}$ RNase A added immediately after incubation. $50 \mu \mathrm{l}$ of $10 \mathrm{nM}$ Tris- $\mathrm{Cl}$ and $0.5 \mathrm{mM}$ EDTA buffer (AE, $\mathrm{pH}$ 9.0) were used for the elution step.

2.2. RNA Extraction. RNA was extracted in an RNase-free environment according to the standard product protocol (RNeasy, Qiagen). All RNA samples were kept on dry ice during extraction. Tissues were lysed using $1.4 \mathrm{~mm}$ ceramic spheres (lysing matrix D, MP Biomedicals, Solon, $\mathrm{OH}$ ) in a tissue homogenizer (Fast Prep 24, MP Biomedicals) and $650 \mu \mathrm{l}$ of lysis buffer (RLT Buffer, Qiagen) with the addition of on-column DNase digestion before RNA purification. $30 \mu \mathrm{l}$ of RNase-free water was used to elute all samples.

2.3. Quantitative Measurements. DNA and RNA quantity (total DNA and RNA) was calculated from concentration multiplied by volume. Concentration was measured using a spectrophotometer (Nanodrop 2000, Thermo Scientific). If the measured ratio of absorbance at $260: 280$ was less than 1.6 for DNA or 1.8 for RNA, the samples were run for an additional time on the chromatography columns in the extraction protocol until the purity threshold was reached.

2.4. Statistical Analysis. The three repeated observations for each tumor sample were averaged into a single observation for analysis after examining the variation of repeated observations using descriptive statistics and graphical displays. Box plots of averaged data were generated for each tumor separately for RNA and DNA and by needle gauge (18G versus $20 \mathrm{G}$ ) and tissue type (normal vs. tumor). For comparisons between tissue type and needle gauge, the Wilcoxon signed-rank test for paired data was used. For comparisons across organ sites (lung versus liver versus kidney), the Kruskal-Wallis rank sum test was applied. A $p$ value $<0.05$ was considered statistically significant. Analyses were conducted using R software version 3.1.0 (R Core Development Team, Vienna, Austria).

2.5. Results. A total of 552 ex vivo biopsies from 46 surgically resected lung $(n=15)$, liver $(n=15)$, and kidney $(n=16)$ specimens were performed. Table 1 indicates the number of biopsies obtained from each organ and the final pathologic diagnosis for each tumor type. The quantitative yield by organ, needle gauge, and tissue type (normal vs. tumor) is provided for DNA and RNA in Tables 2 and 3, respectively. 
Table 1: Pathologic tissue diagnoses by organ.

\begin{tabular}{lc}
\hline Kidney $(n=16)$ & 13 \\
Clear cell carcinoma & 1 \\
Papillary carcinoma & 1 \\
Unclassified renal cell carcinoma & 1 \\
Chromophobe carcinoma & \\
Liver ( $n=15$ ) & 12 \\
Colorectal adenocarcinoma & 1 \\
Hepatocellular carcinoma & 1 \\
Cholangiocarcinoma & 1 \\
Lung adenocarcinoma & \\
Lung ( $n=15$ ) & 6 \\
Squamous cell carcinoma & 5 \\
Lung adenocarcinoma & 1 \\
Carcinoid & 1 \\
Metastatic poorly differentiated carcinoma & 1 \\
Mucinous carcinoma & 1 \\
Lymphoma & $\mathbf{4 6}$ \\
Total number of specimens biopsied & $\mathbf{5 5 2}$ \\
Total biopsy samples (normal + tumor tissues) &
\end{tabular}

2.6. DNA Yield. For all pooled organ sites, the median DNA yield from the larger 18G biopsy needles was significantly greater $(p<0.001)$ than that from $20 \mathrm{G}$ needles in both tumor and normal tissue samples. Median DNA quantities were greater for lung tumor samples compared to normal lung tissue (18G biopsies, $p<0.001$; 20G biopsies, $p<0.001$ ). There was no statistical difference in median DNA obtained from normal versus tumor tissues in the liver or kidney. For all cancer types sampled, the median DNA quantity acquired from single-needle pass $18 \mathrm{G}$ and $20 \mathrm{G}$ core biopsies was $5880 \mathrm{ng}$ (range 1060-13390 ng) and $2710 \mathrm{ng}$ (range 370$6280 \mathrm{ng}$ ), respectively. Box plots in Figure 1 depict median DNA content as well as interquartile ranges for each tissue type and biopsy needle gauge.

2.7. RNA Yield. The median RNA yield from $18 \mathrm{G}$ needles was also significantly greater $(p<0.001)$ than that from 20G biopsies when tumor and normal samples were pooled for all organs. Median RNA quantities were greater for lung tumor tissue compared to nonmalignant tissues for $18 \mathrm{G}$ and $20 \mathrm{G}$ from the lungs (18G biopsies $(p=0.001)$ and $20 \mathrm{G}$ biopsies $(p<0.001)$, respectively) and liver $(p=0.012$ and $p=0.002$, respectively), but not from the kidney. The median RNA quantity from $18 \mathrm{G}$ and $20 \mathrm{G}$ cancer biopsies was $1100 \mathrm{ng}$ (range 110-17210 ng) and $230 \mathrm{ng}$ (range 605210 ng), respectively. Box plots in Figure 2 depict median RNA quantities and interquartile range by needle gauge and tissue type.

\section{Discussion}

In the recent years, cancer genetic technologies such as nextgeneration sequencing (NGS) have evolved, offering insights beyond traditional histopathologic or radiographic diagnoses
[13]. Increased emphasis on molecular characterization has highlighted the role of targeted tissue biopsies in oncology, now routinely obtained for personalized treatment planning and for correlative studies in clinical trials. Gene sequencing for mutation profiling can be particularly challenging for solid tumors as formalin fixatives can disrupt DNA integrity [14]. As nucleic acid yield is not enumerated at the time of biopsy, even when on-site cytopathology review is performed, it can be difficult to determine whether sufficient genetic material has been obtained [15].

While tumor heterogeneity, cellularity, and size as well as other preanalytic parameters and factors can impact downstream analytic success, important information can be gained from studies examining DNA and RNA yield using standardized ex vivo conditions $[16,17]$. Notably, one previous study focused on lung tumor core biopsies reported no statistical difference between in vivo and ex vivo nuclei acid yields within cohorts of the same tumor type [17]. These same authors also attempted to predict tissue yields from core biopsies using needles of different gauges used in clinical practice with multivariate regression. A moderately strong correlation between calculated sampling volume and nucleic acid yield was observed, though analysis was limited to lung tumors and a relatively small number of biopsy samples. In this study, we examined a larger number of primary and metastatic tumor biopsies from the lungs, livers, and kidneys, increasing the potential generalizability of our findings.

Only in the lung, and not in the liver or kidney, did we observe a statistically significant difference in DNA quantities obtained from normal parenchyma versus tumor tissues. In this case, increased cell density, particularly relative to normally air-filled lung tissues, and higher nuclear to cytoplasmic ratios may account for higher quantities of genetic material in lung tumor samples versus normal aerated lung $[18,19]$. RNA differences were observed in the liver and lung but not observed in the kidney. A previous study also reported no difference in RNA content between primary renal malignancies and normal renal parenchyma [20].

Similar to other studies, we found that larger $18 \mathrm{G}$ needles acquired twofold more DNA and fivefold more RNA on average compared to $20 \mathrm{G}$ needles, suggesting that additional needle passes may be necessary to obtain sufficient genetic material when using smaller-gauge needles. The clinical implications of substantial yield variance should not be minimized however, as linear models have not been validated in clinical practice, and smaller $20 \mathrm{G}$ needles can effectively reveal clinically meaningful mutations in lung tumors $[17,21]$. Based on our findings, the notion that one additional large volume core needle pass will guarantee nucleic acid sampling adequacy could lead to analytic failure. In real-world practice, percutaneous biopsy indications, approach, and technique must be considered to minimize procedural morbidity and maximize efficacy. In a recent meta-analysis, the risk for complications following a lung biopsy correlated with larger biopsy needles [22]. Prior knowledge of minimum sampling requirements can facilitate estimation of biopsy feasibility, safety, and likelihood of success. In particular, the number and type of analytic studies to be performed can influence biopsy decisions as higher 
TABle 2: Median DNA (range) from 18- versus 20-gauge needle samples from normal and tumor tissues obtained from the kidney, lung, and liver.

\begin{tabular}{|c|c|c|c|}
\hline & 18G biopsy (ng DNA) & 20G biopsy (ng DNA) & $p$ value (18G vs. $20 \mathrm{G})$ \\
\hline \multicolumn{4}{|l|}{ Any organ } \\
\hline Normal & $4350(1730,13040)$ & $1970(700,5620)$ & $<0.001$ \\
\hline Tumor & $5880(1060,13390)$ & $2710(370,6280)$ & $<0.001$ \\
\hline \multicolumn{4}{|l|}{ Kidney } \\
\hline Normal & $4150(1930,11890)$ & $1360(700,3870)$ & $<0.001$ \\
\hline Tumor & $3170(1180,13390)$ & $1450(370,4600)$ & $<0.001$ \\
\hline$p$ value (normal vs. tumor) & 1.00 & 0.890 & \\
\hline \multicolumn{4}{|l|}{ Lung } \\
\hline Normal & $3240(1740,13040)$ & $1720(760,3520)$ & $<0.001$ \\
\hline Tumor & $6910(3070,12570)$ & $3350(1110,6280)$ & $<0.001$ \\
\hline$p$ value (normal vs. tumor) & $<0.001$ & $<0.001$ & \\
\hline \multicolumn{4}{|l|}{ Liver } \\
\hline Normal & $6050(3790,9740)$ & $2480(1890,5620)$ & $<0.001$ \\
\hline Tumor & $6190(1060,11530)$ & $2630(480,5160)$ & $<0.001$ \\
\hline$p$ value (normal vs. tumor) & 0.804 & 0.847 & \\
\hline
\end{tabular}

TABle 3: Median RNA (range) from 18- versus 20-gauge needle samples from normal and tumor tissues obtained from the kidney, lung, and liver.

\begin{tabular}{|c|c|c|c|}
\hline & 18G biopsy (ng RNA) & 20G biopsy (ng RNA) & $p$ value (18G vs. $20 \mathrm{G}$ ) \\
\hline \multicolumn{4}{|l|}{ Any organ } \\
\hline Normal & $510(30,23540)$ & $240(30,7090)$ & $<0.001$ \\
\hline Tumor & $1100(110,17210)$ & $230(60,5210)$ & $<0.001^{a}$ \\
\hline \multicolumn{4}{|l|}{ Kidney } \\
\hline Normal & $480(230,1210)$ & $270(110,460)$ & $<0.001$ \\
\hline Tumor & $510(220,3420)$ & $290(70,2480)$ & $<0.001$ \\
\hline$p$ value (normal vs. tumor) & 0.855 & 0.217 & \\
\hline \multicolumn{4}{|l|}{ Lung } \\
\hline Normal & $150(30,4940)$ & $120(30,400)$ & 0.008 \\
\hline Tumor & $2870(170,12700)$ & $290(70,2480)$ & $<0.001$ \\
\hline$p$ value (normal vs. tumor) & $<0.001$ & $<0.001$ & \\
\hline \multicolumn{4}{|l|}{ Liver } \\
\hline Normal & $4740(60,23540)$ & $700(60,7090)$ & $<0.001$ \\
\hline Tumor & $1190(110,17210)$ & $150(60,5210)$ & $<0.001$ \\
\hline$p$ value (normal vs. tumor) & 0.012 & 0.002 & \\
\hline
\end{tabular}

${ }^{a}$ Exact test could not be performed due to ties, normal approximation used.

complication rates are associated with increased needle passes and larger-gauge needles $[10,11]$. Ideally, specimen quantities would be well balanced with procedure time and the lowest achievable patient risk.

The practical implications of this study are most apparent in relation to contemporary genetic testing requirements and sources of preanalytic biopsy sample variation. Minimum DNA for NGS can vary depending upon the clinical laboratory technology platform, as well as the target enrichment strategy and number of genes tested in a panel. For example, for the NGS platform currently used at our institution-hybridization capture MiSeq Illumina-based MSK-IMPACT assay [23] - 200-250 ng of DNA is optimally required. DNA quantities as low as $10 \mathrm{ng}$ may be successfully analyzed using the Ion Torrent (Thermo Scientific, Waltham, MA) platform [8], though sequencing errors may occur with limited DNA.

Biopsy sample RNA profiling is highly dependent upon quality, as fragmentation or degradation by RNases can hamper mutational analysis. Microarray technologies can be used to analyze $500 \mathrm{ng}$ of RNA, while NGS and amplification techniques can substantially lower thresholds for clinically meaningful sequencing, even to the level of single-cell genetic material $[24,25]$. 

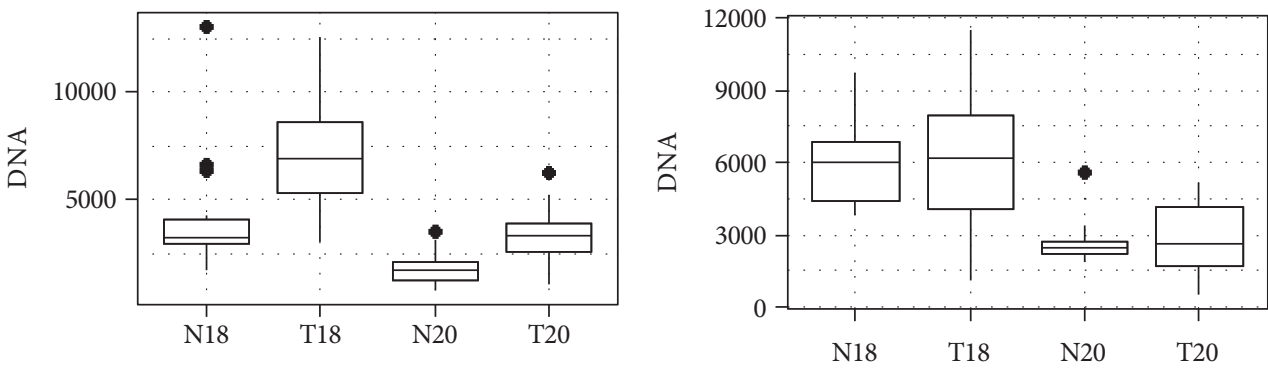

(a)

(b)

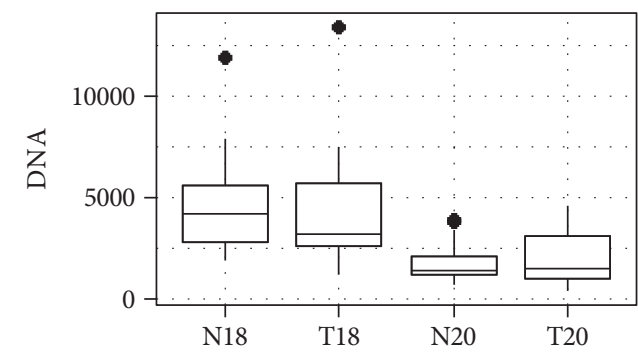

(c)

FIGURE 1: DNA content by tissue type and needle gauge in (a) lung tumors, (b) liver tumors, and (c) kidney tumors (N18= normal tissue/18 gauge, T18 = tumor tissue/18 gauge, N20 = normal tissue/20 gauge, and T20 = tumor tissues/20 gauge). The dark bar represents median DNA quantity, the surrounding box encompasses the $25-75 \%$ interquartile range (IQR), and the brackets reflect $1.5 *$ IQR. Diamonds represent statistical outliers.

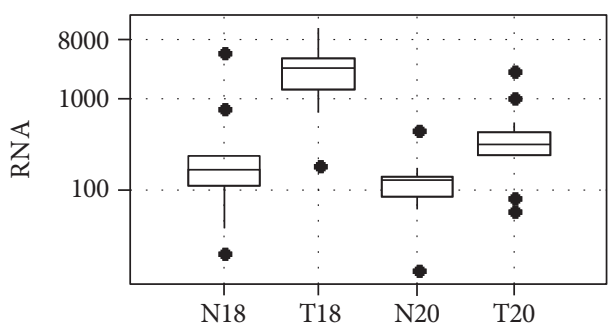

(a)

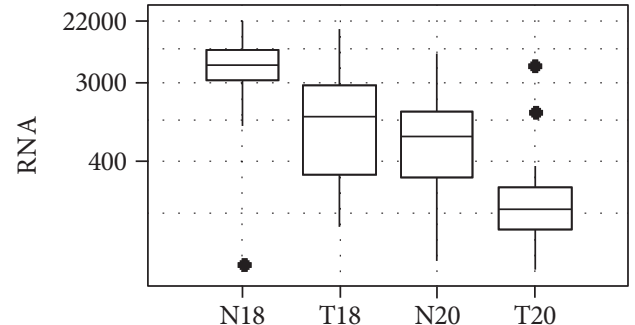

(b)

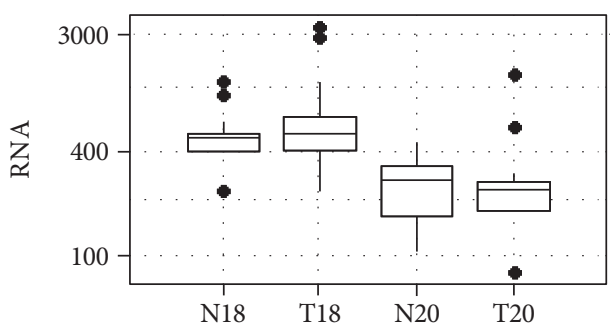

(c)

FIGURE 2: Logarithmic scale of RNA content by tissue type and needle gauge in (a) lung tumors, (b) liver tumors, and (c) kidney tumors $(\mathrm{N} 18=$ normal tissue $/ 18$ gauge, T18 = tumor tissue/18 gauge, $\mathrm{N} 20=$ normal tissue $/ 20$ gauge, T20 $=$ tumor tissues $/ 20$ gauge). The dark bar is the median, the box encompasses the 25-75 interquartile range (IQR), the dotted brackets are $1.5^{*} \mathrm{IQR}$, and dots are outliers.

Assuming no degradation, fragmentation, or other preanalytic disruption of biopsy samples, based on the median DNA content revealed in this study, a single $2 \mathrm{~cm}$ long $18 \mathrm{G}$ or 20G biopsy should be sufficient for most contemporary NGS assays. The same holds true for RNA; however, at the lower end of the RNA range, as low as $60 \mathrm{ng}$ for $20 \mathrm{G}$ core needle biopsies, a single biopsy sample may be more susceptible to analytic failure. In practice, sample degradation and disruption do occur during acquisition and processing. The routine fixation methods fail to conserve the structure of nucleic acids and proteins in tissues. Even short-term treatment of sections with formalin has been shown to significantly reduce the DNA solubility. Similarly, the extraction of useful RNA from FFPE tissue is often compromised 
because of incomplete lysis leading to poor extraction efficiency. In a recent study, RNA extracted from FFPE samples was severely degraded compared to fresh-frozen samples [26]. In our results, the DNA and RNA yield prior to sample processing was 4-6-fold greater than what has previously been reported for FFPE cell blocks [27]. Our results reveal unprocessed nucleic acid yield from core biopsies, which can be frozen at the point of acquisition and submitted for DNA sequencing without FFPE. The potential limitation of this approach is that direct molecular characterization without histopathologic confirmation could result in unconstructive processing of nontumor tissues. Therefore, workflows that increase tumor yield from biopsies, such as radiographic image guidance to confirm needle position within tumor tissues, could mitigate this limitation. Ongoing work suggests that transmission optical spectroscopy imaging of fresh core samples can rapidly characterize tissues at the point of acquisition, which could be used to select appropriate samples for flash freezing prior to molecular diagnostic assays [28].

Although the biopsy yields reported here can serve as a reference for physicians planning or performing molecular studies, the following limitations must be considered in regard to the generalizability of our data. Many factors may reduce the quantity of RNA and DNA suitable for analysis within a small solid tumor biopsy sample, including prior chemotherapy [29] or tumor-associated desmoplasia $[30,31]$. In addition, necrotic tissue has a lower cellular content and can adversely impact biopsy efficacy, even when molecular studies are not planned [32, 33]. While each biopsy was visually screened for a minimum tissue sample length, we did not examine tissues at the microscopic level for cellular composition. We selected tumors that were large enough to fill a $2 \mathrm{~cm}$ core needle biopsy sampling tray. As increased tumor size is associated with increased necrosis [34], we cannot be certain that molecular quantities reported here are valid for larger tumors. By the same token, we cannot certify based on these data that tumors smaller than $2 \mathrm{~cm}$ will contain less genetic material in linear proportion to biopsy sample length. We also used common spectroscopy-based techniques to quantify the molecular content of biopsy samples; however, these types of measurements can result in overestimations. Decreased accuracy is attributed to poor $260: 280 \mathrm{~nm}$ absorption ratios and crosscontamination of RNA and DNA [35]. We used recommended extraction protocols to remove DNA or RNA contaminants; however, remaining contaminants could have influenced yield. Finally, due to the wide variation in the operator technique impacting sampling volumes, we did not study common alternative needle biopsy methods such as fine-needle aspiration.

In summary, we report a wide range of nucleic acid quantities obtained from core needle biopsies in organs commonly afflicted with primary or metastatic cancer. Overall, unprocessed sample nucleic acid quantities are increased relative to FFPE processed tissues; therefore, workflows that bypass fixation and paraffin-based processing may improve yield and utility of core needle sampling for molecular diagnostics.

\section{Data Availability}

The data used to support the findings of this study are available from the corresponding author upon request.

\section{Disclosure}

An earlier version of this work was presented at the 2015 SIR 40th Annual Scientific Meeting.

\section{Conflicts of Interest}

JCD is a member of the Scientific Advisory Board and an investor of Adient Medical and chair of the Society of Interventional Radiology Foundation. SBS is a consultant/advisor at BTG, Johnson \& Johnson, Medtronic, XACT, and Adgero and an investigator at GE Healthcare and AngioDynamics. $\mathrm{He}$ is also an investor of Johnson \& Johnson. JJH is a consultant at Eisai Inc. EZ is an investigator at Ethicon USA, LLC. All other authors have no conflicts of interest.

\section{Acknowledgments}

This research was funded in part through the NIH/NCI Cancer Center Support Grant P30 CA008748. Joanne Chin, MFA, provided editorial assistance with this manuscript.

\section{References}

[1] P. B. Chapman, A. Hauschild, C. Robert et al., "Improved survival with vemurafenib in melanoma with BRAF V600E mutation," The New England Journal of Medicine, vol. 364, no. 26, pp. 2507-2516, 2011.

[2] B. J. Druker, M. Talpaz, D. J. Resta et al., "Efficacy and safety of a specific inhibitor of the BCR-ABL tyrosine kinase in chronic myeloid leukemia," The New England Journal of Medicine, vol. 344, no. 14, pp. 1031-1037, 2001.

[3] D. J. Slamon, B. Leyland-Jones, S. Shak et al., "Use of chemotherapy plus a monoclonal antibody against her2 for metastatic breast cancer that overexpresses HER2," The New England Journal of Medicine, vol. 344, no. 11, pp. 783-792, 2001.

[4] D. Marshall, J. M. Laberge, B. Firetag, T. Miller, and R. K. Kerlan, "The changing face of percutaneous image-guided biopsy: molecular profiling and genomic analysis in current practice," Journal of Vascular and Interventional Radiology, vol. 24, no. 8, pp. 1094-1103, 2013.

[5] S. Gupta, M. J. Wallace, J. F. Cardella et al., "Quality improvement guidelines for percutaneous needle biopsy," Journal of Vascular and Interventional Radiology, vol. 21, no. 7, pp. 969-975, 2010.

[6] A. L. Tam, H. J. Lim, I. I. Wistuba et al., "Image-guided biopsy in the era of personalized cancer care: proceedings from the Society of Interventional Radiology Research Consensus Panel," Journal of Vascular and Interventional Radiology, vol. 27, no. 1, pp. 8-19, 2016.

[7] A. Sankin, A. A. Hakimi, N. Mikkilineni et al., "The impact of genetic heterogeneity on biomarker development in kidney cancer assessed by multiregional sampling," Cancer Medicine, vol. 3, no. 6, pp. 1485-1492, 2014.

[8] H. Chen, R. Luthra, R. S. Goswami, R. R. Singh, and S. Roy-Chowdhuri, "Analysis of pre-analytic factors 
affecting the success of clinical next-generation sequencing of solid organ malignancies," Cancers, vol. 7, no. 3, pp. 16991715, 2015.

[9] S. Roy-Chowdhuri, R. S. Goswami, H. Chen et al., "Factors affecting the success of next-generation sequencing in cytology specimens," Cancer Cytopathology, vol. 123, no. 11, pp. 659668, 2015.

[10] P. R. Geraghty, S. T. Kee, G. McFarlane, M. K. Razavi, D. Y. Sze, and M. D. Dake, "CT-guided transthoracic needle aspiration biopsy of pulmonary nodules: needle size and pneumothorax rate," Radiology, vol. 229, no. 2, pp. 475-481, 2003.

[11] G. R. Vijayaraghavan, S. Vedantham, V. Rangan et al., "Effect of needle gauge and lobe laterality on parenchymal liver biopsy outcome: a retrospective analysis," Abdominal Imaging, vol. 40, no. 5, pp. 1223-1229, 2015.

[12] K. M. Corapi, J. L. T. Chen, E. M. Balk, and C. E. Gordon, "Bleeding complications of native kidney biopsy: a systematic review and meta-analysis," American Journal of Kidney Diseases, vol. 60, no. 1, pp. 62-73, 2012.

[13] J. N. McCutcheon and G. Giaccone, "Next-generation sequencing: targeting targeted therapies," Clinical Cancer Research, vol. 21, no. 16, pp. 3584-3585, 2015.

[14] M. Srinivasan, D. Sedmak, and S. Jewell, "Effect of fixatives and tissue processing on the content and integrity of nucleic acids," The American Journal of Pathology, vol. 161, no. 6, pp. 19611971, 2002.

[15] R. L. Schmidt, B. L. Witt, L. E. Lopez-Calderon, and L. J. Layfield, "The influence of rapid onsite evaluation on the adequacy rate of fine-needle aspiration cytology: a systematic review and meta-analysis," American Journal of Clinical Pathology, vol. 139, no. 3, pp. 300-308, 2013.

[16] M. C. Austin, C. Smith, C. C. Pritchard, and J. F. Tait, "DNA yield from tissue samples in surgical pathology and minimum tissue requirements for molecular testing," Archives of Pathology \& Laboratory Medicine, vol. 140, no. 2, pp. 130-133, 2016.

[17] N. Jamshidi, D. Huang, F. G. Abtin et al., "Genomic adequacy from solid tumor core needle biopsies of ex vivo tissue and in vivo lung masses: prospective study," Radiology, vol. 282, no. 3, pp. 903-912, 2016.

[18] S. A. Fuhrman, L. C. Lasky, and C. Limas, "Prognostic significance of morphologic parameters in renal cell carcinoma," American Journal of Surgical Pathology, vol. 6, no. 7, pp. 655-664, 1982.

[19] T. Muto, H. J. R. Bussey, and B. C. Morson, "The evolution of cancer of the colon and rectum," Cancer, vol. 36, no. 6, pp. 2251-2270, 1975.

[20] J. J. Elser, M. M. Kyle, M. S. Smith, and J. D. Nagy, "Biological stoichiometry in human cancer," PLoS One, vol. 2, no. 10, article e1028, 2007.

[21] S. B. Solomon, M. F. Zakowski, W. Pao et al., "Core needle lung biopsy specimens: adequacy for EGFR and KRAS mutational analysis," American Journal of Roentgenology, vol. 194, no. 1, pp. 266-269, 2010.

[22] W. J. Heerink, G. H. de Bock, G. J. de Jonge, H. J. M. Groen, R. Vliegenthart, and M. Oudkerk, "Complication rates of CT-guided transthoracic lung biopsy: meta-analysis," European Radiology, vol. 27, no. 1, pp. 138-148, 2017.

[23] D. T. Cheng, T. N. Mitchell, A. Zehir et al., "Memorial Sloan Kettering-Integrated Mutation Profiling of Actionable cancer Targets (MSK-IMPACT): a hybridization capture-based next-generation sequencing clinical assay for solid tumor molecular oncology," The Journal of Molecular Diagnostics, vol. 17, no. 3, pp. 251-264, 2015.

[24] J. Shendure and H. Ji, "Next-generation DNA sequencing," Nature Biotechnology, vol. 26, no. 10, pp. 1135-1145, 2008.

[25] M. L. Metzker, "Sequencing technologies-the next generation," Nature Reviews. Genetics, vol. 11, no. 1, pp. 31-46, 2010.

[26] J. Li, C. Fu, T. P. Speed, W. Wang, and W. F. Symmans, "Accurate RNA sequencing from formalin-fixed cancer tissue to represent high-quality transcriptome from frozen tissue," JCO Precision Oncology, vol. 2018, no. 2, pp. 1-9, 2018.

[27] S. K. Tian, J. K. Killian, N. Rekhtman et al., "Optimizing workflows and processing of cytologic samples for comprehensive analysis by next-generation sequencing: Memorial Sloan Kettering Cancer Center experience," Archives of Pathology \& Laboratory Medicine, vol. 140, no. 11, pp. 1200-1205, 2016.

[28] M. T. Silk, S. Yazdanfar, D. V. Dylov, T. Silk, S. B. Solomon, and J. C. Durack, "Rapid determination of core needle biopsy adequacy using optical spectroscopy," in SIR 39th annual scientific meeting, p. S117, San Diego, CA, USA, 2014.

[29] R. R. White, H. B. Xie, M. R. Gottfried et al., "Significance of histological response to preoperative chemoradiotherapy for pancreatic cancer," Annals of Surgical Oncology, vol. 12, no. 3, pp. 214-221, 2005.

[30] R. Kalluri and M. Zeisberg, "Fibroblasts in cancer," Nature Reviews. Cancer, vol. 6, no. 5, pp. 392-401, 2006.

[31] R. Kadaba, H. Birke, J. Wang et al., "Imbalance of desmoplastic stromal cell numbers drives aggressive cancer processes," The Journal of Pathology, vol. 230, no. 1, pp. 107-117, 2013.

[32] J. F. Pan, P. C. Yang, D. B. Chang, Y. C. Lee, S. H. Kuo, and K. T. Luh, "Needle aspiration biopsy of malignant lung masses with necrotic centers: improved sensitivity with ultrasonic guidance," Chest, vol. 103, no. 5, pp. 1452-1456, 1993.

[33] S. Sartori, I. Nielsen, L. Trevisani, P. Tombesi, P. Ceccotti, and V. Abbasciano, "Contrast-enhanced sonography as guidance for transthoracic biopsy of a peripheral lung lesion with large necrotic areas," Journal of Ultrasound in Medicine, vol. 23, no. 1, pp. 133-136, 2004.

[34] K. De Jaeger, F. M. Merlo, M.-C. Kavanagh, A. W. Fyles, D. Hedley, and R. P. Hill, "Heterogeneity of tumor oxygenation: relationship to tumor necrosis, tumor size, and metastasis," International Journal of Radiation Oncology, Biology, Physics, vol. 42, no. 4, pp. 717-721, 1998.

[35] Thermo Scientific, "260/280 and 260/230 Ratios," T009-Technical Bulletin, pp. 1-2, 2008, http://www.nhm.ac.uk/content/ dam/nhmwww/our-science/dpts-facilities-staff/Coreresearch labs/nanodrop.pdf. 


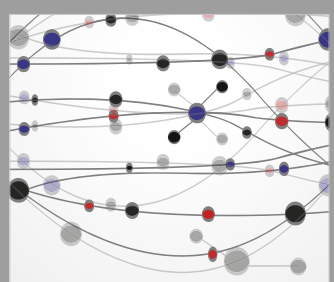

The Scientific World Journal
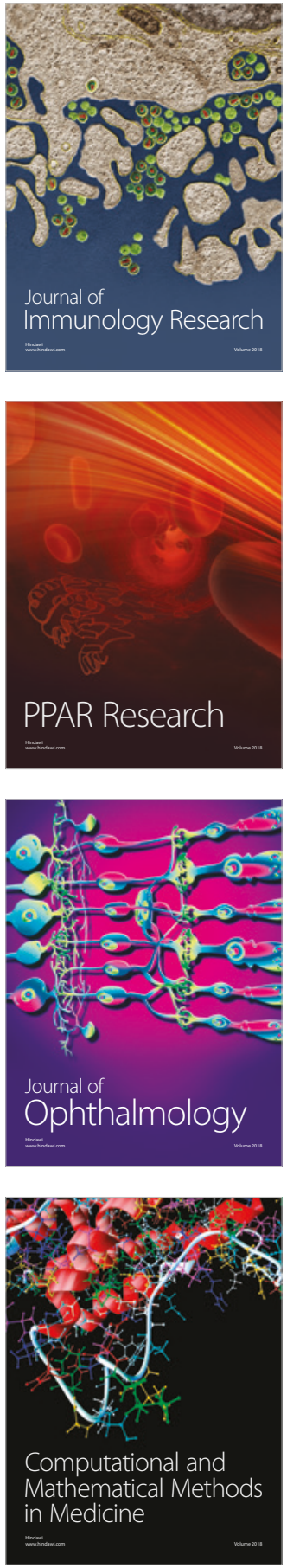

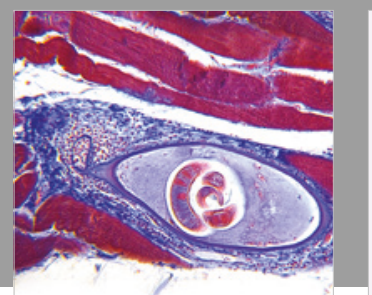

Gastroenterology Research and Practice

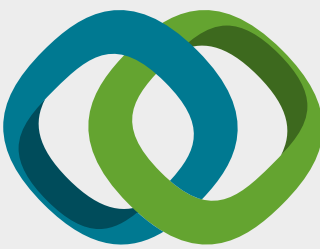

\section{Hindawi}

Submit your manuscripts at

www.hindawi.com
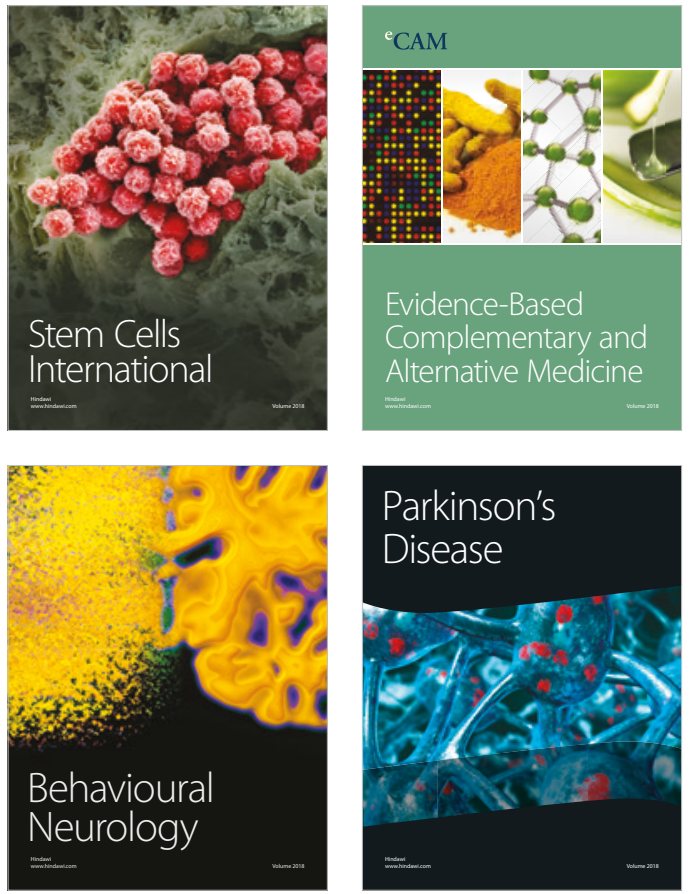

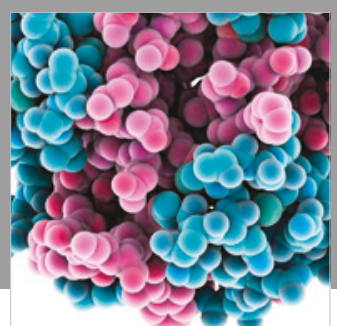

ournal of

Diabetes Research

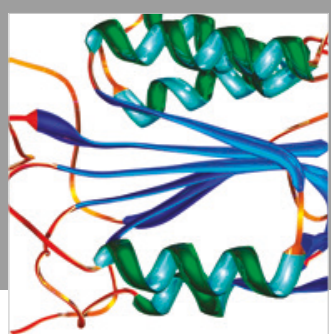

Disease Markers
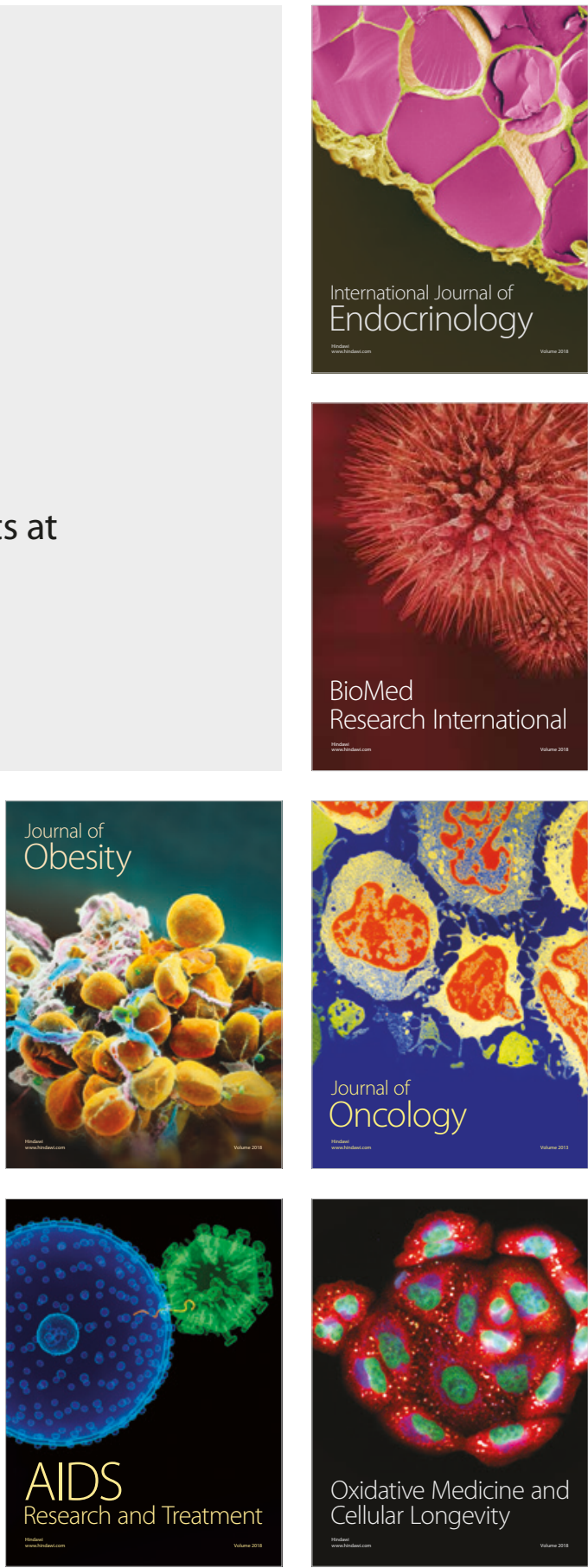